\title{
PROPOSTA DE NOVA METODOLOGIA DE ENSINO PARA A ÁREA DE MANUFATURA NO CURSO DE ENGENHARIA MECÂNICA
}

DOI: 10.37702/2175-957X.COBENGE.2021.3559

Luciana Montanari - montanar@sc.usp.br

Universidade de São Paulo

Av. Trabalhador São-carlense 400

13566-590 - São Carlos - SP

Alessandro Roger Rodrigues - roger@sc.usp.br

Universidade de São Paulo

Av. Trabalhador São-Carlense 400

13566-590 - São Carlos - SP

arthur jose vieira porto - ajvporto@sc.usp.br

UNIVERSIDADE DE SAO PAULO

rua virgilio pozzi 120

13564-040 - sao carlos - SP

Resumo: Trata este artigo de uma proposta de reformulação das unidades curriculares da Área de Manufatura, do Curso de Graduação em Engenharia Mecânica, da Escola de Engenharia de São Carlos (EESC), Universidade de São Paulo (USP). Ao currículo acadêmico original, formatado em 6 unidades curriculares e estruturado por processo de fabricação, propõe-se sua substituição por 4 unidades curriculares, e fundamentado pela geometria do produto a ser fabricado e requisitos de qualidade, aliados a questões de custo, sustentabilidade e projeto da manufatura. Além da reformulação curricular em termos de quantidade e objeto das unidades curriculares, novas metodologias de ensino-aprendizagem e avaliação são propostas, baseadas na maior participação discente e menor protagonismo docente, tais como lab-makers, blended learning, problem-based learning, team-based learning, avaliação formativa, autoavaliação e avaliação entre pares. Espera-se com esta nova proposta uma aprendizagem de conhecimentos teórico-práticos mais ativa e construtivista por parte dos alunos, fornecendo-Ihes habilidades e competências demandadas pela engenharia de fabricação atual no Brasil e no mundo, com atualizações tecnológicas incorporadas mais dinamicamente, como sustentabilidade e Indústria 4.0 e 5.0. 


\section{COBENCE - Everno onine

Palavras-chave: Engenharia Mecânica. Manufatura. Reformulação curricular. Metodologias ativas. Processos avaliativos. 


\section{PROPOSTA DE NOVA METODOLOGIA DE ENSINO PARA A ÁREA DE MANUFATURA NO CURSO DE ENGENHARIA MECÂNICA}

\section{INTRODUÇÃO}

A primeira turma de engenheiros formados no curso de Engenharia Mecânica da Escola de Engenharia de São Carlos da Universidade de São Paulo (EESC-USP) foi em 1957. A área de Projeto e Manufatura sempre teve destaque no currículo do curso devido à própria constituição do Departamento à época. Professores conceituados como Dino Ferraresi, Carlos Pallerosi e Horst Dar compunham o quadro docente. Eles empregavam metodologias de ensino-aprendizagem que haviam aprendido com os seus professores e as suas experiências, ademais, eram pertinentes de acordo com o perfil do estudante do curso, do mercado de trabalho, do contexto da Universidade na região e do ambiente de aprendizagem.

No entanto, esse cenário foi alterado. São Carlos atualmente possui uma diversidade de indústrias, especialmente voltadas à tecnologia, devido à concentração de doutores formados e atuantes (AGÊNCIA FAPESP, 2019; FONTES, 2019). Os estudantes são da chamada Geração Z (MCCRINDLE, 2014) e a EESC cresceu e se fortaleceu. Há vários convênios que facilitam a experiência internacional de professores e alunos, e o mercado de trabalho nacional visa se inserir no contexto da Indústria 4.0, enquanto parte do mercado internacional tem em vista a Indústria 5.0 (SKOBELEV e BOROVIK, 2017). Além disso, os desafios impostos pela pandemia da COVID 19 contribuíram para a inclusão de novas metodologias de ensino e a aceleração do ensino digital.

Para a implantação da Indústria 4.0 no mercado nacional, foram destacados, segundo a Confederação Nacional da Indústria ( $\mathrm{CNI})$, sete desafios com dimensões prioritárias: i) aplicações nas cadeias produtivas e desenvolvimento de fornecedores; ii) mecanismos para induzir a adoção das novas tecnologias; iii) desenvolvimento tecnológico; iv) ampliação e melhoria da infraestrutura de banda larga; v) aspectos regulatórios; vi) formação de recursos humanos; e vii) articulação institucional (CNI, 2016).

Em 2020, a CNI realizou um estudo sobre a difusão das tecnologias da Indústria 4.0 em empresas brasileiras e concluiu que há uma dicotomia na relação entre a engenharia de produto e a engenharia de manufatura. Os produtos são idealizados e desenhados independente do processo de manufatura. $\mathrm{E}$ entende que seria razoável pensar que 0 desenvolvimento do processo ocorresse conjuntamente com o desenvolvimento do produto. Dessa forma, a manufaturabilidade do produto, que pudesse conceber a forma mais econômica e eficiente de produção, não é devidamente considerada pela engenharia (CNI, 2020).

Em paralelo aos avanços tecnológicos, mas em sentido oposto, encontram-se as alterações realizadas na estrutura do ensino básico, no qual o ensino de conteúdos tecnológicos, especificamente os voltados ao desenho descritivo e técnico, foram suprimidos dos currículos escolares, tendo como consequência direta o fato de os estudantes apresentarem dificuldades na visualização espacial de objetos tridimensionais.

A perda pela supressão do desenho técnico no ensino básico potencializa a dificuldade do ensino e do despertar do interesse pela manufatura, pois o primeiro contato dos alunos com a realidade é complexa beleza da manufatura dava-se ao desenhar as primeiras peças e, por consequência, advinham as perguntas "Para que serve?", "Como 
usar?" e "Como fabricar?". E, como um dos pilares da Manufatura 4.0 é o emprego de modelos topológicos, faz-se necessário suprir estas deficiências do ensino básico.

Nesse contexto, uma oportunidade para reformular o ensino da manufatura se apresentou no âmbito da EESC-USP, onde está ocorrendo um processo de modernização e atualização dos currículos de seus cursos, instado pela Direção da Unidade Acadêmica, descrito no Projeto Acadêmico da EESC para o período compreendido entre 2019 e 2023 (EESC, 2019) e pelas novas diretrizes curriculares para os cursos de engenharia (BRASIL, 2019). Este processo de modernização está sendo norteado por algumas diretrizes: diminuição do número de horas-aula expositivas, utilização de novas técnicas, metodologias e ferramentas de ensino, atualização do conhecimento essencial que se necessita ensinar em cada área de concentração respeitando o perfil do egresso almejado pela sociedade.

Para a reformulação do ensino na área de manufatura no curso de Engenharia Mecânica da EESC-USP, o ponto de partida teve como base as competências declaradas no perfil do egresso do Projeto Pedagógico do Curso (PPC) que visam atender o mercado nacional, internacional e a sociedade. Dentre elas destacam-se: a resolução de problemas complexos, o pensamento crítico, a capacidade de interação de diferentes áreas do conhecimento, a capacidade de julgamento e a tomada de decisões, a capacidade de absorver e desenvolver novas tecnologias, aprender de forma autônoma e lidar com situações e contextos complexos, atualizando-se em relação aos avanços da ciência e tecnologia (EESC, 2020).

Isto posto, o objetivo deste trabalho é apresentar uma proposta de um novo conjunto de unidades curriculares e metodologias de ensino na área de manufatura, que alie competências técnicas essenciais e interpessoais dos futuros engenheiros às novas dinâmicas de ensino-aprendizagem, no que diz respeito ao emprego de lab-makers, ensino híbrido e metodologias ativas. Também são propostas novas formas e dinâmicas de avaliação, como avaliação progressiva, autoavaliação, avaliação entre pares, avaliação formativa e debates.

\section{PROPOSTA}

Um olhar para o passado sobre o ensino da manufatura no curso de Engenharia Mecânica da EESC-USP mostra um ensino centrado no funcionamento das máquinasferramentas. Uma proposta de modernização do ensino, baseada em novas metodologias de ensino e avaliação, seria o ensino centrado na produção das peças, de forma otimizada com controle de fluxo, qualidade e custo.

\subsection{0 currículo antigo}

O ensino da manufatura pouco mudou desde o início do curso. A abordagem para a formação do aluno nessa área consiste em, através de um conjunto de unidades curriculares expositivas e algumas aulas demonstrativas, apresentar os conceitos básicos dos processos de fabricação, seguidos dos modelos matemáticos e, então, alguns exemplos. A motivação parte do princípio do entendimento do processo, com foco no funcionamento das máquinas-ferramentas. As unidades curriculares da área de Manufatura do curso de Engenharia Mecânica da EESC são apresentadas no "Quadro 1". 
28 a 30 de SETEMBRO

Quadro 1 - Unidades curriculares da área de Manufatura do curso de Engenharia Mecânica EESC-USP

\begin{tabular}{|c|c|}
\hline Unidade curricular & Principais tópicos abordados \\
\hline Usinagem dos Metais & $\begin{array}{c}\text { Mecanismo da formação dos cavacos, materiais e mecanismos de } \\
\text { desgaste de ferramentas de corte, forças de corte, potência de } \\
\text { corte, temperatura de corte, fluidos de corte e economia da } \\
\text { usinagem. }\end{array}$ \\
\hline Processos de Usinagem & $\begin{array}{c}\text { Torneamento, fresamento, furação, roscamento, mandrilamento, } \\
\text { alargamento, retificação. }\end{array}$ \\
\hline $\begin{array}{c}\text { Fundamentos da Fabricação } \\
\text { Mecânica }\end{array}$ & $\begin{array}{c}\text { Sistemas de referência, análise de dimensões, sequência de } \\
\text { fabricação, planos de processo. }\end{array}$ \\
\hline $\begin{array}{c}\text { Processos de Conformação e } \\
\text { Não Convencionais }\end{array}$ & $\begin{array}{c}\text { Introdução à teoria da plasticidade, processos de: corte, dobra, } \\
\text { estampagem, forjamento, laminação, soldagem, eletroerosão, feixe } \\
\text { de elétrons, laser, plasma. }\end{array}$ \\
\hline $\begin{array}{c}\text { Manufatura Assistida por } \\
\text { Computador }\end{array}$ & $\begin{array}{c}\text { Sistemas de coordenadas, programação CNC manual, } \\
\text { programação CAM, robôs industriais, programação robótica, } \\
\text { arranjos físicos industriais, sistemas de manufatura, movimentação } \\
\text { de materiais. }\end{array}$ \\
\hline $\begin{array}{c}\text { Práticas em Processos de } \\
\text { Fabricação Mecânica }\end{array}$ & $\begin{array}{c}\text { Práticas de torneamento, fresamento, furação, retificação, } \\
\text { estampagem e corte. }\end{array}$ \\
\hline
\end{tabular}

Fonte: USP (2008)

Imagens e gráficos são comumente usados como ferramentas de ensino para auxiliar na explicação sobre o funcionamento dos processos de fabricação. Os alunos muitas vezes ficam entediados na sala de aula quando muitas imagens e gráficos são apresentados diversas vezes para ilustrar os detalhes dos diferentes processos de fabricação. Na unidade curricular de Práticas em Processos de Fabricação Mecânica são realizadas demonstrações do funcionamento e capabilidade de algumas máquinasferramentas, já em Mecânica são Processos de Usinagem e Manufatura Assistida por Computador, a critério e iniciativa do docente, são realizadas algumas visitas técnicas e aplicação da metodologia de ensino baseado em problemas, respectivamente. Entretanto, na maioria das unidades curriculares as aulas são expositivas, com o desenvolvimento de exercícios teóricos e projetos de aplicação, tais como o desenvolvimento de plano de processo e de código $\mathrm{G}$.

A estrutura de unidades curriculares do currículo antigo proporciona um ensino compartimentado e estanque, de tal forma que os alunos somente adquirirão a competência necessária para a manufatura de peças após a realização de toda a sequência dos cursos, sem a oportunidade de exercitar a correlação dos conhecimentos, provocando uma perda no aprendizado.

\subsection{A proposta}

A essência que caracteriza esta proposta é a mudança do foco do conjunto de unidades curriculares para a "geração das peças" e o emprego de novas metodologias de ensino-aprendizagem e métodos de avaliação. Observando a "Figura 1", entende-se que o foco é a geração de peças. Dessa forma, é possível conceber que o desenvolvimento do processo ocorre conjuntamente com o desenvolvimento do produto, mostrando ao estudante que a manufaturabilidade do produto deve conceber a forma mais econômica e eficiente de produção. 
Figura 1 - Concepção da proposta.

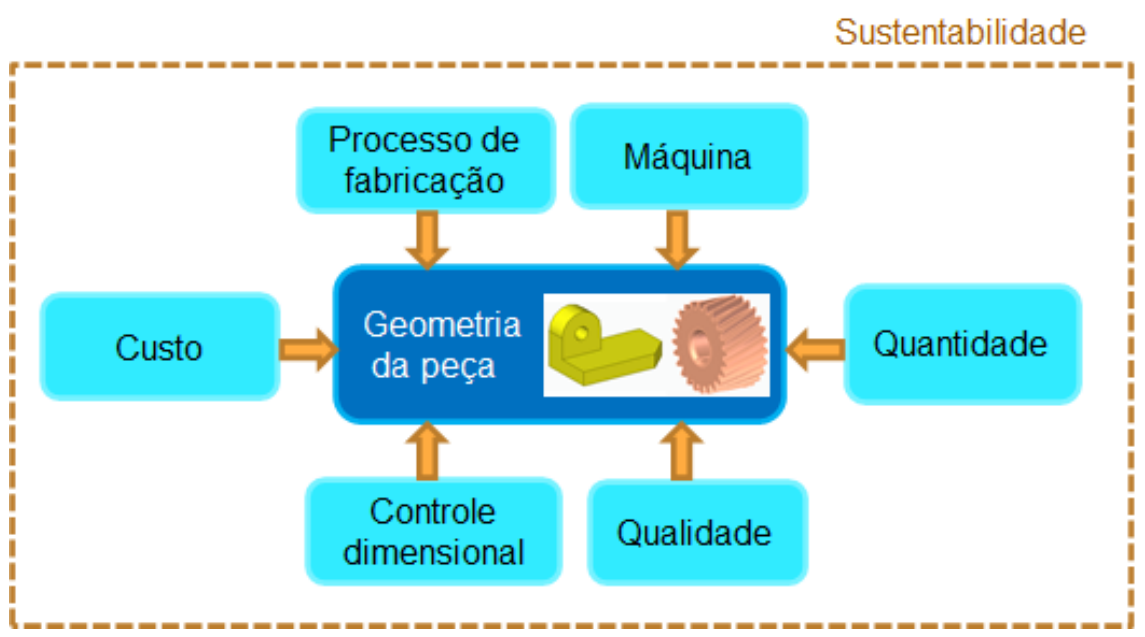

Fonte: Próprios autores

Uma busca em alguns tradicionais cursos de Engenharia Mecânica internacionais, corroborou o enfoque na geração da peça, sendo que alguns apresentam também uma abordagem no projeto e na gestão do processo produtivo.

O novo conjunto de unidades curriculares ("Quadro 2") foi planejado para o ensino do conteúdo essencial em manufatura, tendo como objetivo ensinar a produzir uma peça ao invés de ensinar a fabricar uma superfície. É importante que um estudante de engenharia seja capaz de selecionar a tecnologia mais adequada para a fabricação de uma determinada geometria.

Quadro 2 - Novo conjunto de unidades curriculares

\begin{tabular}{|c|c|c|}
\hline Unidade curricular & Principais tópicos abordados & Objetivos gerais \\
\hline $\begin{array}{l}\text { Introdução à } \\
\text { Manufatura }\end{array}$ & $\begin{array}{l}\text { Fundamentos dos processos de fabricação, } \\
\text { conceitos de projeto visando seleção do } \\
\text { material e processo, conceitos de fabricação } \\
\text { baseados em máquinas, materiais das } \\
\text { peças, metrologia e montagem, importância } \\
\text { da metrologia na inspeção de peças, } \\
\text { instrumentos de metrologia, conexão entre } \\
\text { processos considerando aspectos de } \\
\text { fabricação econômica, funcional e } \\
\text { sustentabilidade. }\end{array}$ & $\begin{array}{c}\text { - descrever os processos de } \\
\text { fabricação de acordo com o } \\
\text { projeto de peças } \\
\text { - } \quad \text { relacionar tópicos de } \\
\text { metrologia e montagem }\end{array}$ \\
\hline $\begin{array}{c}\text { Manufatura por } \\
\text { Processos } \\
\text { Subtrativos }\end{array}$ & $\begin{array}{c}\text { Processos de remoção dos materiais } \\
\text { (usinagem), ferramentas e métodos de corte } \\
\text { da peça, tipos de fixação da peça, tipos de } \\
\text { máquinas, operações de referência, } \\
\text { sobremetal de usinagem, tratamentos } \\
\text { térmicos e produtividade, aplicação em } \\
\text { peças rotacionais e peças prismáticas, } \\
\text { métodos de controle dimensional, de fluxo e } \\
\text { qualidade, arranjos de fábrica e } \\
\text { apresentação das técnicas de custeio, } \\
\text { sustentabilidade. }\end{array}$ & $\begin{array}{c}\text { A partir de uma peça com dada } \\
\text { geometria (prismática ou } \\
\text { rotacional): } \\
\text { - diferenciar como os } \\
\text { processos de fabricação } \\
\text { subtrativos podem ser } \\
\text { empregados } \\
\text { - discutir, analisar e } \\
\text { estabelecer os métodos de } \\
\text { medição de peça e } \\
\text { especificação das tolerâncias } \\
\text { dimensionais e geométricas } \\
\text { - aplicar conceitos de } \\
\text { sustentabilidade }\end{array}$ \\
\hline
\end{tabular}




\begin{tabular}{|c|c|c|}
\hline $\begin{array}{l}\text { Fabricação por } \\
\text { Processos Aditivos } \\
\text { e Conformativos }\end{array}$ & $\begin{array}{c}\text { Processos de adição (solda, impressão 3D), } \\
\text { processos conformativos (forjamento a } \\
\text { quente e a frio, estampagem, corte e } \\
\text { injeção), aplicação em peças rotacionais e } \\
\text { peças prismáticas, controle dimensional, de } \\
\text { fluxo e qualidade, arranjos de fábrica e } \\
\text { apresentação das técnicas de custeio, } \\
\text { sustentabilidade. }\end{array}$ & $\begin{array}{c}\text { A partir de uma peça com dada } \\
\text { geometria (prismática ou } \\
\text { rotacional): } \\
\text { - diferenciar como os } \\
\text { processos de fabricação } \\
\text { aditivos e conformativos } \\
\text { podem ser empregados } \\
\text { - discutir, analisar e } \\
\text { estabelecer os métodos de } \\
\text { medição de peça e } \\
\text { especificação das tolerâncias } \\
\text { dimensionais e geométricas } \\
\text { - aplicar conceitos de } \\
\text { sustentabilidade }\end{array}$ \\
\hline $\begin{array}{l}\text { Sistemas de } \\
\text { Manufatura }\end{array}$ & $\begin{array}{l}\text { Arranjos físicos, modelos de controle de } \\
\text { produção, filosofia de produção }\end{array}$ & $\begin{array}{c}\text { - relacionar o planejamento do } \\
\text { processo de fabricação das } \\
\text { peças e a dependência da } \\
\text { geometria da peça, } \\
\text { equipamentos disponíveis, } \\
\text { lote e qualidade da peça. } \\
\text { - combinar processos de } \\
\text { fabricação para a produção } \\
\text { de peças } \\
\text { - avaliar as tolerâncias } \\
\text { dimensionais e geométricas } \\
\text { em uma sequência de } \\
\text { fabricação }\end{array}$ \\
\hline
\end{tabular}

Fonte: Próprios autores

A estrutura de cada unidade curricular da proposta visa proporcionar um ensino integrador do conhecimento, fornecendo aos alunos todos os conhecimentos necessários para a manufatura dos tipos de peças tratados no contexto da unidade curricular, com ênfase no oferecimento de oportunidade para o exercício aplicado dos conhecimentos no próprio seio da unidade curricular, garantindo um ganho do aprendizado.

\section{ANÁLISE CRÍTICA DA PROPOSTA}

Selecionar tópicos apropriados e conectá-los de uma forma sistemática, lógica e atrativa para cada unidade curricular por si só é um desafio. No entanto, a esse desafio soma-se a necessidade de obter, como resultado da aprendizagem, um profissional capaz de atender a sociedade e o mercado. Por isso, é necessário estabelecer metodologias de ensino e processos de avaliação que contribuam para a formação dos estudantes. As principais metodologias que serão aplicadas são: Problem-Based Learning (PBL), TeamBased Learning (TBL), Ensino Híbrido, Sala de Aula Invertida, Lab-Makers, "gamificação" como já indicado no "Quadro 2".

Os processos de avaliação que deverão ser empregados serão escolhidos entre: Avaliação Formativa, Autoavaliação, Avaliação entre Pares, Debates, Avaliação de trabalhos em grupo ou uma composição entre eles. No "Quadro 3" destacam-se alguns 
métodos de avaliação que contribuirão com o processo de ensino/aprendizagem já indicados para cada unidade curricular.

Quadro 3 - Relação entre disciplina e processo de avaliação

\begin{tabular}{|c|c|}
\hline Unidade curricular & Avaliação \\
\hline Introdução à Manufatura & Autoavaliação e debates \\
\hline Manufatura por Processos Subtrativos & Avaliação formativa e entre pares \\
\hline $\begin{array}{c}\text { Fabricação por Processos Aditivos e } \\
\text { Conformativos }\end{array}$ & $\begin{array}{c}\text { Avaliação formativa e avaliação de } \\
\text { trabalhos em grupo }\end{array}$ \\
\hline Sistemas de Manufatura & $\begin{array}{c}\text { Avaliação formativa e avaliação de } \\
\text { trabalhos em grupo. As atividades em } \\
\text { grupo poderão ser baseadas na } \\
\text { "gamificação" }\end{array}$ \\
\hline
\end{tabular}

Fonte: Próprios autores

Para contribuir com o processo de ensino-aprendizagem, propõe-se, além das metodologias de ensino, enfoque do conteúdo e processo avaliativo, o desenvolvimento de um repositório para abrigar mídias desenvolvidas para exemplificar a manufatura de peças diversas e também mídias comerciais acrescidas de comentários e análises técnicas realizados pelos docentes.

O uso de laboratórios virtuais como elementos de apoio ao sistema de ensinoaprendizagem deverá ser incentivado ou obrigatório, principalmente para os tópicos de conhecimento dependentes da aplicação de softwares específicos, tais como no ensino de Manufatura Assistida por Computador (geração de código G) e no Projeto via Simulação de Sistemas de Manufatura (modelo dinâmico do sistema de manufatura). Portanto, o ensino híbrido surge como uma estratégia para complementar as metodologias de ensino/aprendizagem.

Visando estudar a viabilidade de implantação do novo conjunto de unidades curriculares, algumas das metodologias de ensino propostas para as unidades curriculares apresentadas no "Quadro 2" foram aplicadas durante o período de pandemia. Por exemplo, para a unidade curricular Manufatura Assistida por Computador ("Quadro 1"), dada a impossibilidade de aulas práticas presenciais, os alunos utilizaram simuladores de máquinas-ferramentas CNC (Comando Numérico Computadorizado) open-source disponíveis na internet. Tais simuladores permitiram testar os programas CNC elaborados pelos alunos manualmente ou via programas CAM (Manufatura Assistida por Computador) em ambientes realistas, quanto à operação da máquina CNC, fixação da peça, setup das ferramentas, testes de colisão ferramenta-peça-sistemas de fixação e etapas da usinagem.

Além disso, antes da pandemia, por iniciativas particulares de alguns docentes, foram aplicadas algumas metodologias ativas de ensino como, por exemplo, aprendizado baseado em problemas e visitas técnicas.

A "Figura 2" mostra algumas atividades realizadas nas unidades curriculares Processos de Usinagem e Manufatura Assistida por Computador apresentadas no "Quadro 1 " antes da pandemia.

Figura 2 - Atividades realizadas nas unidades curriculares antes da pandemia: (a) Processos de Usinagem e (b) Manufatura Assistida por Computador. 


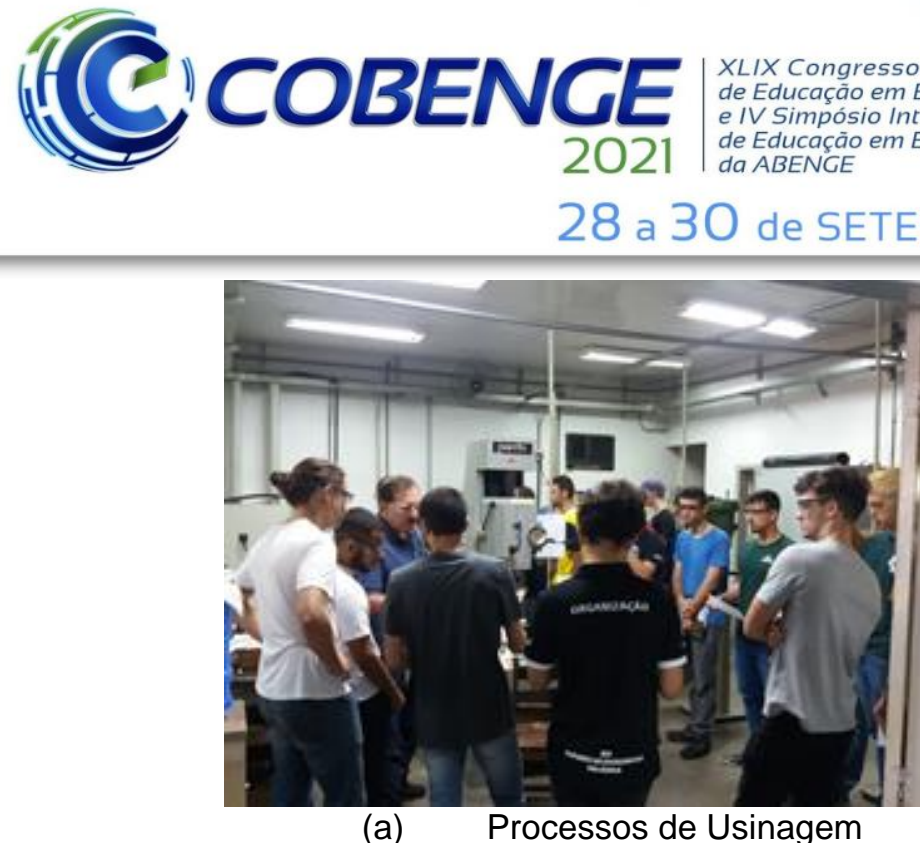

Fonte: Próprios autores
- Evento Online

"Formação em Engenharia:

Tecnologia, Inovação e Sustentabilidade"

A "Figura 2(a)" apresenta uma imagem de uma aula demonstrativa, cujo objetivo era observar a sequência de fabricação de uma peça que envolvia os processos de fresamento, furação, roscamento, mandrilamento e alargamento. Já a "Figura 2(b)" mostra as equipes reunidas discutindo o projeto e usinagem de peças fabricadas por softwares CAD/CAM (Projeto Assistido por Computador/Manufatura Assistida por Computador).

$\mathrm{Na}$ "Figura 3" são apresentadas fotos registradas antes da pandemia, durante as visitas técnicas realizadas em empresas renomadas no Brasil e no exterior voltadas ao projeto e fabricação de máquinas-ferramentas CNC (Indústrias Romi S.A.) e aviação (Embraer S.A.). As visitas técnicas contribuem com a formação dos estudantes no sentido de promover o elo entre a teoria e a prática "in loco", motivando-os e favorecendo a fixação de conceitos com a vivência em diferentes ambientes de aprendizagem.

Figura 3 - Visitação nas Indústrias Romi S.A. (a) e Embraer S.A. (b).

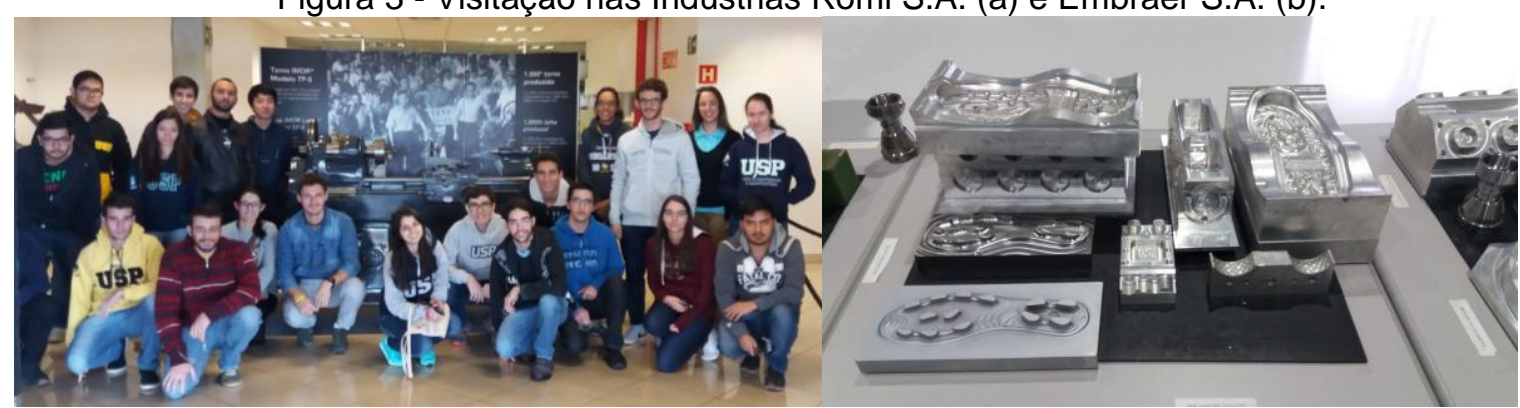

(a) Indústrias Romi S.A.
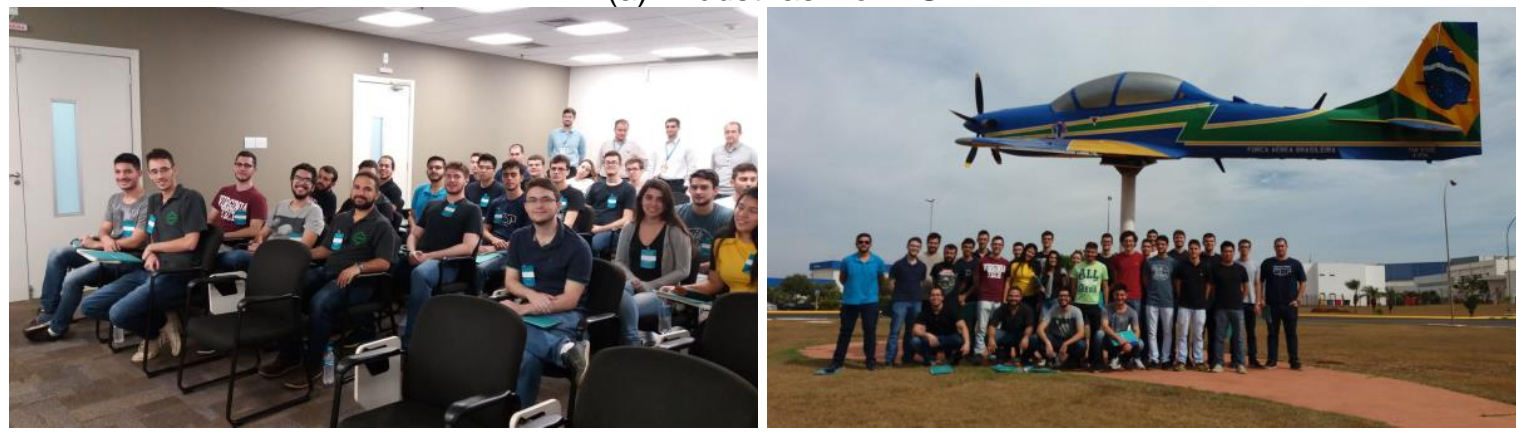

(b) Embraer S.A.

Fonte: Próprios autores

O resultado positivo dessas iniciativas particulares de alguns docentes que, independentemente da ementa, implantaram metodologias ativas de ensino em algumas 
unidades curriculares, antes e durante a pandemia, corrobora e reforça a necessidade de mudança do ensino na área de Manufatura do curso de Engenharia Mecânica da EESCUSP.

Desse modo, a reunião entre os objetivos de cada unidade curricular, as metodologias de ensino e os processos de avaliação permitirão buscar as transformações digitais que compõem as bases da Indústria 4.0, ou seja, o uso intensivo de redes e plataformas digitais integradas, softwares aplicados (CAD, CAM, Planejamento do Processo, Simulação de Sistemas, Planejamento de Fábrica etc.) e interoperabilidade de dados (dados da peça, requisitos da produção, dados da fábrica etc.) permitirão a iniciação dos alunos nos conceitos da Indústria 4.0.

\section{CONSIDERAÇÕES FINAIS}

As unidades curriculares do currículo antigo utilizadas para o ensino da manufatura proporcionam um ensino compartimentado e estanque, de tal forma que os alunos somente adquirirão a competência necessária para a manufatura de peças após a realização de toda a sequência da cadeia. Esse método de ensino mostrou-se não atrativo aos alunos e a perda de interesse pela área é refletida, por exemplo, na baixa procura por trabalhos em Projeto Final de Curso e em Pós-graduação nessa área. Após esta constatação, buscouse por uma proposta em que cada unidade curricular proporcionasse um ensino integrado de conhecimento, com ênfase no oferecimento de oportunidade para o exercício aplicado dos conhecimentos no cerne da unidade curricular, garantindo um ganho do aprendizado.

Concomitante a este novo conjunto de disciplinas, buscou-se por novas metodologias de ensino, algumas já experimentadas com êxito, que possibilitassem o ganho de aprendizado através de atividades de integração e aplicação dos conhecimentos realizadas durante a disciplina, sendo que estas atividades também seriam integradoras do processo de avaliação da disciplina, pois poderão ser realizadas de modo contínuo e progressivo, até que o aluno ou equipe de alunos atinjam o resultado esperado para a atividade.

\section{REFERÊNCIAS}

AGÊNCIA FAPESP (Fundação de Amparo do Estado de São Paulo). São Carlos tem um doutor a cada 100 habitantes, São Paulo, 24 mai. 2019. Disponível em: <https://agencia.fapesp.br/sao-carlos-tem-um-doutor-a-cada-100-habitantes/30594/>. Acesso em: 29 abr. 2021.

BRASIL (Ministério da Educação). Resolução CES/CNE/MEC n.ำ 2, de 24 de abril de 2019. Dispõe sobre diretrizes curriculares nacionais do curso de graduação em engenharia. Disponível em: <https://www.in.gov.br/web/dou/-/resoluÇÃo-n-o-2-de-24-deabril-de-2019-85344528>. Acesso em: 9 de ago. 2020.

CNI (Confederação Nacional da Indústria). Desafios para a indústria 4.0 no Brasil, Brasília: CNI, 34 p., 2016. Disponível em:

$<$ https://static.portaldaindustria.com.br/media/filer public/d6/cb/d6cbfbba-4d7e-43a0-978486365061a366/desafios para industria 40 no brasil.pdf>. Acesso em: 5 maio 2021.

CNI (Confederação Nacional da Indústria). A difusão das tecnologias da indústria 4.0 em empresas brasileiras, Brasília: CNI, 54 p., 2016. Disponível em: 
<https://static.portaldaindustria.com.br/media/filer public/c4/26/c42635b7-c3c0-47638ed2-69aa33b8a07e/a difusao das tecnologias da industria 40 vf.pdf $>$. Acesso em: 5 maio 2021.

EESC (Escola de Engenharia de São Carlos). Projeto Acadêmico 2019-2023, 33p., 2019. Disponível em:

<http://www.jaguar.eesc.usp.br/portaleesc/images/docs/eesc projeto academico.pdf>. Acesso em: 5 maio 2021.

EESC (Escola de Engenharia de São Carlos). Projeto Pedagógico Curso Engenharia Mecânica, 2020. Disponível em: <https://eesc.usp.br/graduacao/curso.php?id=18062>. Acesso em: 3 maio 2021.

FONTES, H. Cidade de São Carlos tem um doutor a cada 100 habitantes. Jornal da USP, São Paulo, 14 abr. 2019. Disponível em:

$<$ https://jornal.usp.br/universidade/cidade-de-sao-carlos-tem-um-doutor-a-cada-100habitantes/>. Acesso em: 29 abr. 2021.

MCCRINDLE, M. The ABC of XYZ: In: Understanding the Global Generations. 2014. The generation map. Disponível em: <https://2qean3b1jjd1s87812ool5ji-wpengine.netdnassl.com/wp-content/uploads/2016/12/McCrindle-Research_ABC-03_The-GenerationMap_Mark-McCrindle.pdf>. Acesso em: 29 abr. 2021.

SKOBELEV, P.O.; BOROVIK, S.YU. On the way from industry 4.0 to industry 5.0: from digital manufacturing to digital society. International Scientific Journal "Industry 4.0", n. 6, p. 307-311, 2017. Disponível em <https://stumejournals.com/journals/i4/2017/6/307.full.pdf>. Acesso em: 5 maio 2021.

USP (Universidade de São Paulo). Informações da disciplina, 2008. Disponível em: $<$ https://uspdigital.usp.br/jupiterweb/jupDisciplinaBusca?tipo=D\&codmnu=6755>. Acesso em 5 maio 2021.

\title{
PROPOSAL OF A NEW LEARNING METHODOLOGY FOR MANUFACTURING AREA IN MECHANICAL ENGINEERING UNDERGRADUATE COURSE
}

\begin{abstract}
This paper presents a proposal of curricular reformulation for the Manufacturing Area of the Mechanical Engineering Undergraduate Course, at São Carlos School of Engineering (EESC), University of São Paulo (USP). To the original academic curriculum, which was designed in 6 curricular units and structured by manufacturing processes, it is proposed its substitution by 4 curriculum units now based on the product geometry to be manufactured and quality requirements, combined with costs issues, sustainability and manufacturing design. In addition to the curricular improvement in terms of quantity and object of curricular units, modern teaching-learning methodologies, as well as assessment processes are also proposed aiming more student participation and less professor protagonism, such as lab-makers, blended learning, problem-based learning, team-based learning, formative assessment, self-assessment, peer-assessment. It is expected with this
\end{abstract}


new approach more active and constructivist learning of theoretical-practical knowledge by students so that they reach competences and abilities which are demanded by the current manufacturing engineering scenery in Brazil and around the world by considering technological improvements dynamically added such as sustainability and Industry 4.0 and 5.0.

Keywords: Mechanical Engineering. Manufacturing. Curriculum reformulation. Active methodologies. Assessment processes. 